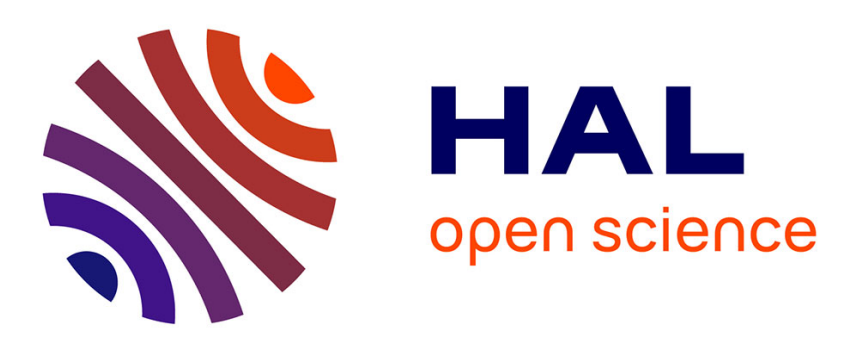

\title{
Two-Phase Displacement In Porous Media Studied By MRI Techniques
}

Jamal Fannir, Irina Panfilov, Sébastien Leclerc, Didier Stemmelen, Sebastien

Leclerc

\section{- To cite this version:}

Jamal Fannir, Irina Panfilov, Sébastien Leclerc, Didier Stemmelen, Sebastien Leclerc. Two-Phase Displacement In Porous Media Studied By MRI Techniques. 16th European Conference on the Mathematics of Oil Recovery (ECMOR XVI), Sep 2018, Barcelone, Spain. 10.3997/2214-4609.201802188 . hal-01883685v2

\section{HAL Id: hal-01883685 \\ https://hal.univ-lorraine.fr/hal-01883685v2}

Submitted on 2 May 2019

HAL is a multi-disciplinary open access archive for the deposit and dissemination of scientific research documents, whether they are published or not. The documents may come from teaching and research institutions in France or abroad, or from public or private research centers.
L'archive ouverte pluridisciplinaire HAL, est destinée au dépôt et à la diffusion de documents scientifiques de niveau recherche, publiés ou non, émanant des établissements d'enseignement et de recherche français ou étrangers, des laboratoires publics ou privés. 


\title{
Two-phase displacement in porous media studied by MRI techniques
}

\author{
Jamal Fannir, Irina Panfilov, Sébastien Leclerc, Didier Stemmelen \\ LEMTA (UMR 7563 - Université de Lorraine), 54504 VANDOEUVRE-LES-NANCY, FRANCE
}

\section{Introduction}

The study and modeling of two-phase flow in porous media is of considerable interest in the petroleum industry (estimation of exploitable reserve, optimization of the recovery techniques...) but also in the sector of chemical industry (catalytic reactors, separation, and extraction) or in the hydrogeology domain (pollution of aquifers by NAPL). This explains the importance of the studies aiming to improve the description of multi-phase flow in porous media. Notably, the simultaneous flow of two immiscible fluids (water-oil) in porous media is not always well described by Darcy's generalized law, which only takes into account water/oil saturation as additional descriptive variable. This has a substantial impact on the modeling of the two-phase flow stability under the simultaneous effect of the force of gravity, viscosity and superficial tension.

For the study of two-phase flow in porous media, several kinds of visualization methods have been applied including X-ray, computed tomography scanners (CT) and magnetic resonance imaging (MRI) techniques. Among these methods, only MRI is able to visualize the internal structure of a system in three dimensions with a spatial resolution of the species present. Furthermore, MRI also allows in-situ measurements of flow velocity, saturation and diffusion dispersion of the fluid flow within the porous media. Another opportunity of the MRI is that it can be used to probe both microscopic and macroscopic properties.

The rapid development of new quantitative magnetic resonance imaging technologies led to new opportunities with interesting results for measurements of fluid flow in porous media. Johns and Gladden (1998) have used MRI technique to visualize the dissolution of entrapped ganglia or "blobs" of octanol within the pore space of a randomly packed bed of glass beads, by a mobile aqueous phase. They also acquired three dimensional images and were able to distinguish the solid, hydrocarbon, and aqueous phases. Finally, they also obtained velocity maps of the mobile aqueous phase. Ersland et al. (2010) used the MRI techniques to study how the oil recovery is affected by fractures. They have obtained high spatial resolution images of the water-oil flow inside a $1 \mathrm{~mm}$ fracture. Furthermore, in a recent work in LEMTA, MRI has been employed for studies of fluid flow within granular porous media (Salameh et al. 2010). The authors have shown the possibility of visualization of the velocity field in porous media and accurate measurements of interstitial and averaged velocities in packed beds.

\section{Research objective and methodology}

The purpose of the present study is the investigation of the immiscible fluid displacement in porous medium between wetting and non-wetting fluids as an important factor for secondary recovery of hydrocarbons. Furthermore, several types of forces such as capillary, viscous and gravitational are extremely important to describe the two-phase flow. In this project, MRI was used to experimentally examine the water-oil flux through a vertical porous model.

Using MRI technique, we also study the dynamics of the displacement front, its deformation and the phase trapping during the two-phase flow process. Displacement in porous media is characterized by the formation of different structures of the phase distribution in porous space. For the same saturation, the fluids can form different shapes. So these various shapes can be described by the displacing phase saturation $S$ and an additional parameter, responsible for the type of the shape. It is not sufficient to only have the specific surface of phase distribution in space, as proposed by several authors, because different shapes can have a surface size of the same order. We recommend using two additional 
parameters of shape: one to characterize the specific interface between the phases, and the other to evaluate the degree of connectivity between the phases.

The saturation of the displacing phase, the connected displaced phase and disconnected traps, and also the menisci distribution in the zone of the front deformation allow us to complete the phenomenological menisci model proposed in (Panfilova and Panfilov 2005). Moreover, a numerical simulation of the two-phase flow in the vertical tube was performed and the results were compared to the experimental ones.

\section{Experimental}

To perform the experimental study, we use an MRI spectrometer equipped with a $14 \mathrm{~T}$ magnet (corresponding to $1 \mathrm{H}$ resonance frequency of $600 \mathrm{MHz}$ ). With this technique we measure and visualize directly the two phase flow in a vertical model of porous medium (packed beads or sands) under the ambient condition. The MRI visualization technique allows for non-invasive measurements (2D or even 3D mapping) of the concentration of hydrogen nuclei in the liquid phase in the porous media. These hydrogen nuclei can belong to molecules of water or other liquids. The $1 \mathrm{H}$ species associated with water or hydrocarbon provide the phase distribution images, and it is also possible to distinguish the solid and liquid phases.

A scheme of the experimental setup is shown in Fig.1. The setup consists of three parts: the first one is $600 \mathrm{MHz}$ MRI spectrometer, for which the available diameter of the imaging probe is $40 \mathrm{~mm}$. The second part is the porous medium column. We have used two different types of materials for tubes and beads according to their wettability. A tube with $34 \mathrm{~mm}$ internal diameter and $14 \mathrm{~cm}$ in height was filled with polystyrene (or borosilicate) beads of $0.6 \mathrm{~mm}$ diameter. The beads are kept inside the tube by two porous filters and two caps. A syringe pump is connected to the system to inject the water upward. This pump is able to inject at a very low rate with an accuracy of $\pm 0.5 \%$. The third part is the computer control to monitor of the MRI data signal.

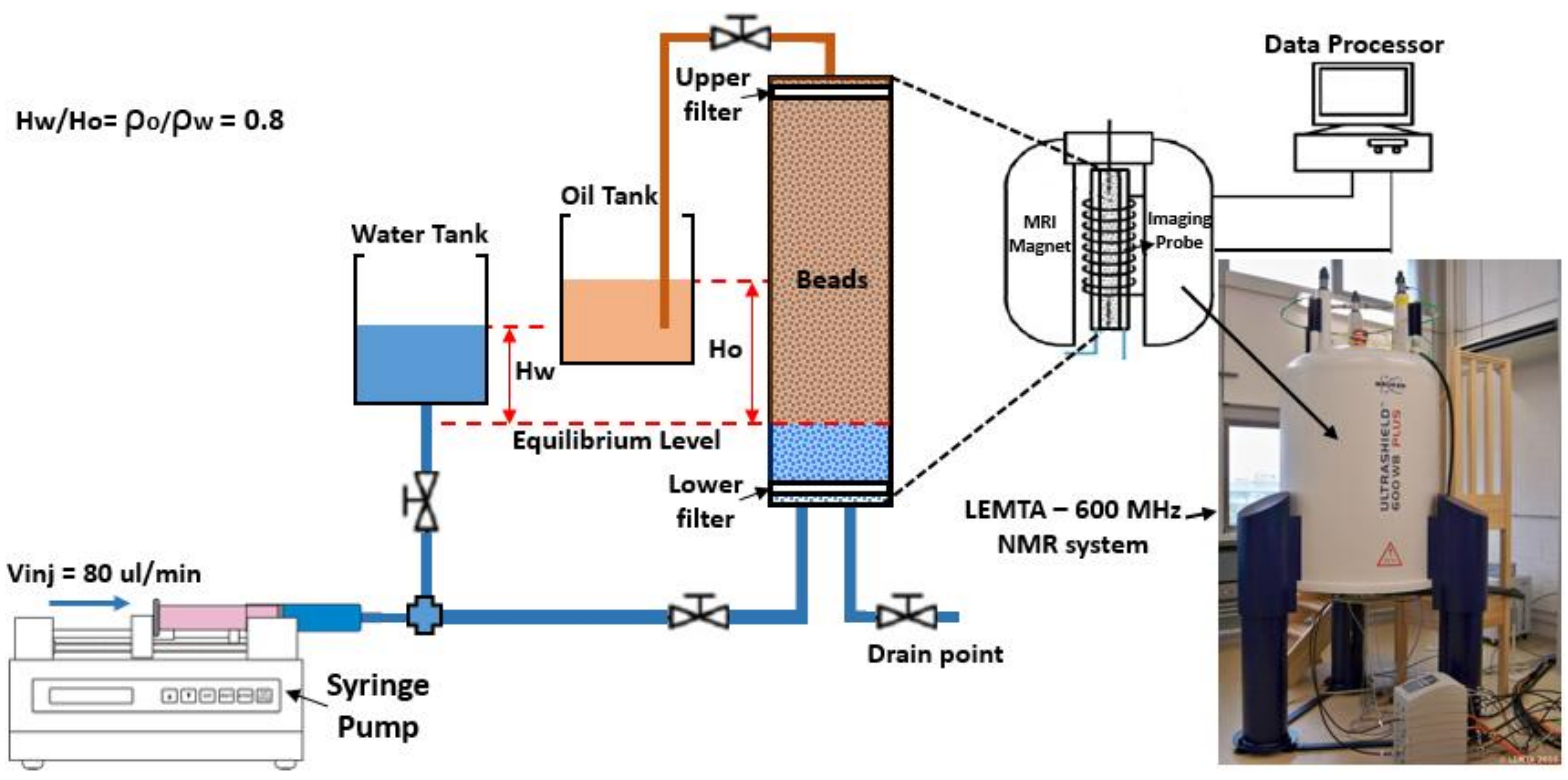

Figure 1: Experimental setup.

To saturate the sample, a vacuum pump is first used to remove air. This way, we obtained fully saturated samples. At initial state, we install a hydrostatic equilibrium of water-oil contact inside of the porous sample which is calculated with the levels of water and oil in tanks. Doing this the water oil interface is homogeneously distributed near the bottom filter. The injection rate was controlled by the syringe pump. The water was pumped into the column from the bottom with the objective to prevent the deformation of water-oil interface by the gravity. The volumetric flowrate was varied 
from 0.04 to $2 \mathrm{ml} / \mathrm{min}$, the corresponding superficial velocity was calculated using the geometry and porosity of model. The tuning and matching of the probe were adjusted before acquiring each image. The probe adjustment stopped when the sweeping of oil was finished and no change was observed on the tuning parameters.

For each experiment the MR images were taken during more than 10 hours, a period corresponding to the injection of 3 and more pore volumes of water. Table 1 shows the main parameters of oil phase displacement when a plastic tube and polystyrene beads were used.

\begin{tabular}{ll}
\hline Table 1. Water flooding experiment in plastic-bead pack \\
\hline Porous & Polystyrene beads of $~ 0.6 \mathrm{~mm}$ diameter \\
\hline Tube & Plastic tube of $10 \mathrm{~cm}$ net length \\
\hline Injection rate & $0.12 \mathrm{ml} / \mathrm{min}$ \\
\hline Estimated porosity & $38 \%$ \\
\hline Injected fluid & Pure water $+\mathrm{MnCl}_{2}(\mathrm{C}=0.01 \mathrm{~mole} / \mathrm{l})$ \\
\hline Oil phase (kerosene) & PTX2000 $\rho=800 \mathrm{~kg} / \mathrm{m3}, \mu=2.96 \mathrm{mPa}$ s at $20^{\circ} \mathrm{C}$ \\
MRI Field of view & $5 \mathrm{~cm}(256 \times 256 \mathrm{pixels})$ \\
\hline TR (time of repetition) & $1 \mathrm{~s}$ \\
\hline TE (time of echo) & $15 \mathrm{~ms}$ \\
\hline
\end{tabular}

\section{Nonselective method}

Successive trials were performed with the objective to obtain high quality MRI images. The first model used was characterized by a PVC tube packed with polystyrene beads of $0.6 \mathrm{~mm}$ diameter. Some water is injected into the model, initially fully saturated by oil, to displace the fluid. The contrast between species (immiscible hydrocarbon and aqueous phases) in MRI experiments is usually achieved by using a chemical shift selective imaging method (Mitchell et al. 2013). The first results were satisfactory and we were able to observe the internal structure of the medium (Fig.2).

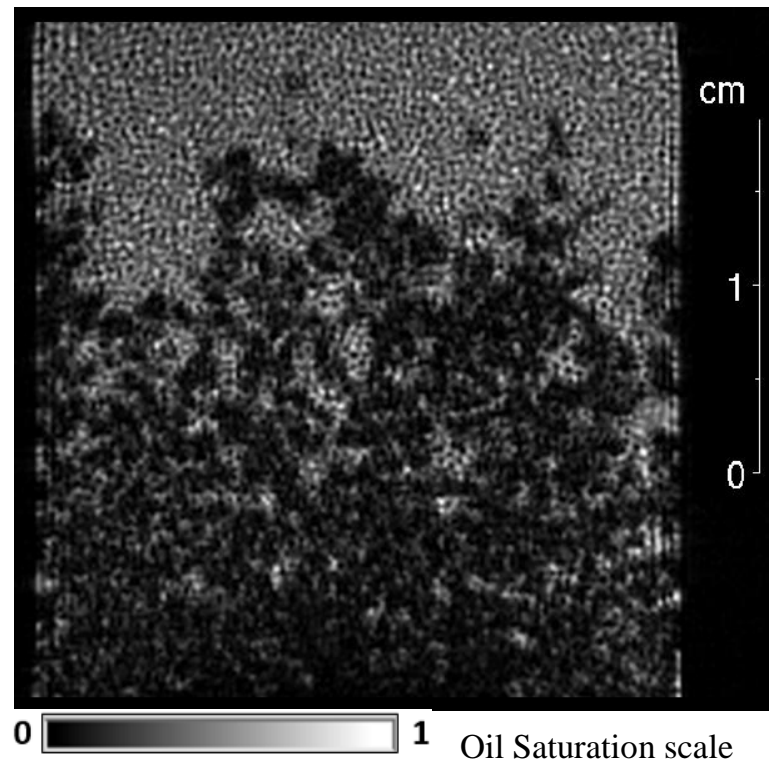

$a$

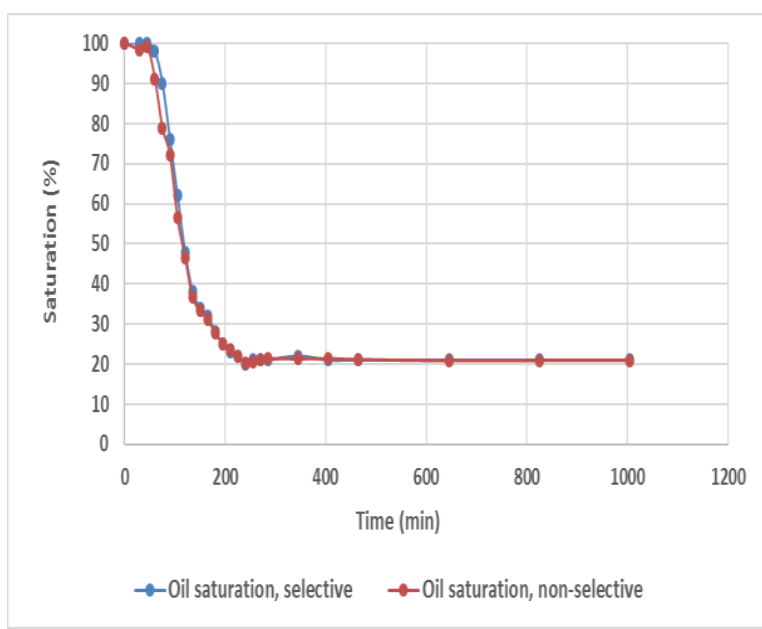

$b$

Figure 2: MRI image of polystyrene beads of 0.6mm stacked in PVC tube and saturated initially with oil after 3 h45 of water injection with the rate of $0.12 \mathrm{ml} / \mathrm{min}(\mathrm{a})$; saturation profile of oil phase during displacement for selective and non-selective methods $(b)$.

In spite of the fact that the polystyrene beads and the tube have a quasi-zero signal, the interface between oil and water was deformed by chemical shift artifacts. A number of methods were 
implemented to avoid this effect, namely non-selective imagery, modification of the intensity of the magnetic field gradient etc. Finally, the solution was in "the cancelling" of the water signal. For biological applications of Magnetic Resonance, Bloembergen (1957) has found that the addition on $\mathrm{Mn}^{2+}$ affected the relaxation decay of the water phase ( $\mathrm{T}_{2}$ of water can be shorted to below $\left.1 \mathrm{~ms}\right)$. Instead of water, we used an aqueous solution of $\mathrm{MnCl} 2$ with a very low $\mathrm{T}_{2}$ time. With such a short relaxation time, the signal of water disappear so quickly that it is not possible to image it. However, as $\mathrm{MnCl} 2$ is not soluble in organic compounds, there is no effect on the relaxation time of oil $(\sim 1000$ $\mathrm{ms})$. Therefore, the signal of oil is the only measured signal.

The non-selective method with $\mathrm{MnCl}_{2}$ solution in water give clear images of oil trapping for a $\mathrm{FOV}$ (field of view) of $5 \mathrm{~cm}$ (Fig.2a). The NS-method also shows the good coincidence with the selective technique (Fig.2b). The obtained images were satisfactory from the point of view of high spatial resolution $(0.19 \mathrm{~mm}$ for 1 pixel) and visualization of the dynamic evolution of the two-phase.

\section{Experiment with a packed polystyrene beads model}

The raw images obtained are processed with Matlab software to obtain the visualization of the dynamic process of displacement of the oil (kerosene) by the injected aqueous solution (water + manganese chloride). These images have a resolution of $256 \times 256$ pixels for a field of view (FOV) of $5 \mathrm{~cm}$. For each model, data are recorded on vertical and horizontal sections with an interval of 15 minutes between each series of images. The recorded data are then processed with Matlab to retrieve the images on which statistical analyzes can be done.

Fig. 3 shows the consecutive series of images, reproduced with Matlab in vertical and horizontal directions, for the experiment of the polystyrene beads model with the injection flow rate of 0.12 $\mathrm{ml} / \mathrm{min}$.

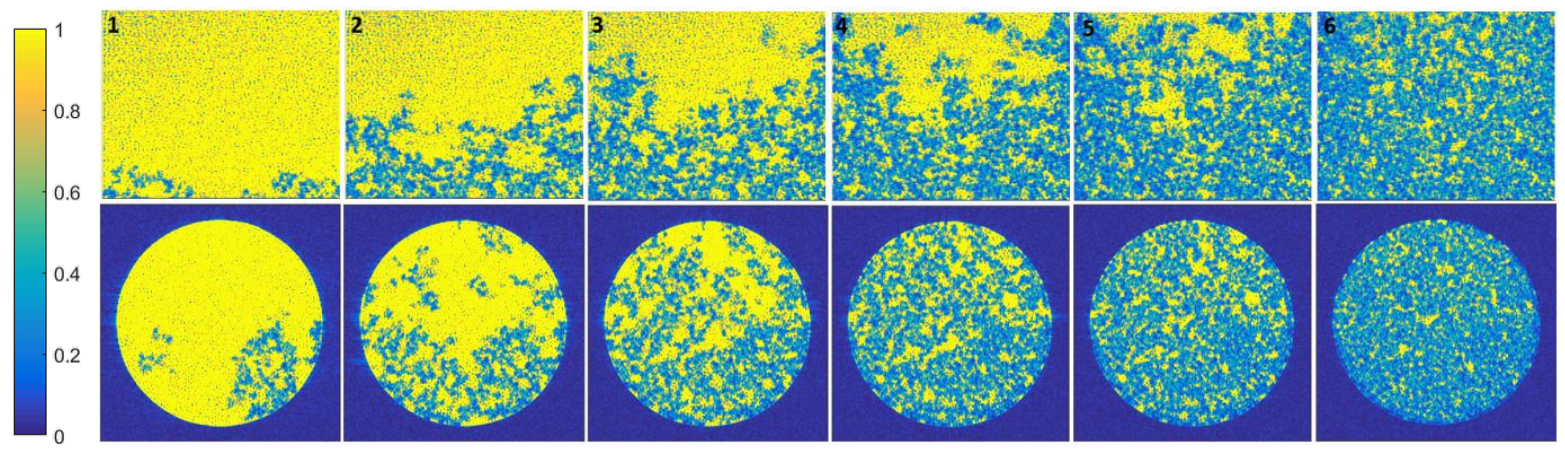

Figure 3: Consecutive MRI images of the water-oil displacement in the model of polystyrene beads with the injection flow rate of $0.12 \mathrm{ml} / \mathrm{min}$. The acquisition of MRI signal was made each 15 minutes.

We note that the displacement of the oil by water does not correspond to a piston-like flow, but generates digits leading to a trapping of the oil during the two-phase flow. At the end of the displacement process the total residual oil saturation, averaged through the sample volume, is equal to $38 \%$. Indeed, the residual oil saturation obtained at the end of the process is an average saturation throughout the model, while it is observed that the saturation is not uniformly distributed. It is therefore necessary to observe in detail what happens at each position along of the model.

The analysis of the last image (Fig.4), where the oil saturation was stabilized, shows an increasing distribution of the residual oil saturation as a function of the vertical position. However, it is easy to observe that the residual oil saturation is much greater in the highest sections of the model than in the lowest sections, these differences are due to the development of digitations/fingerings along the model. We also note that the continuous field of low oil saturation, observed in Figure 4(a) in light blue color, corresponds to the oil film left on the surface of the polystyrene beads. The variation of saturation of these zones depends on the thickness of these films and the interactions between oil, 
water and surface of the beads. Thus, a distinction was made between these oil films and the oil trapped due to the development of digitations.
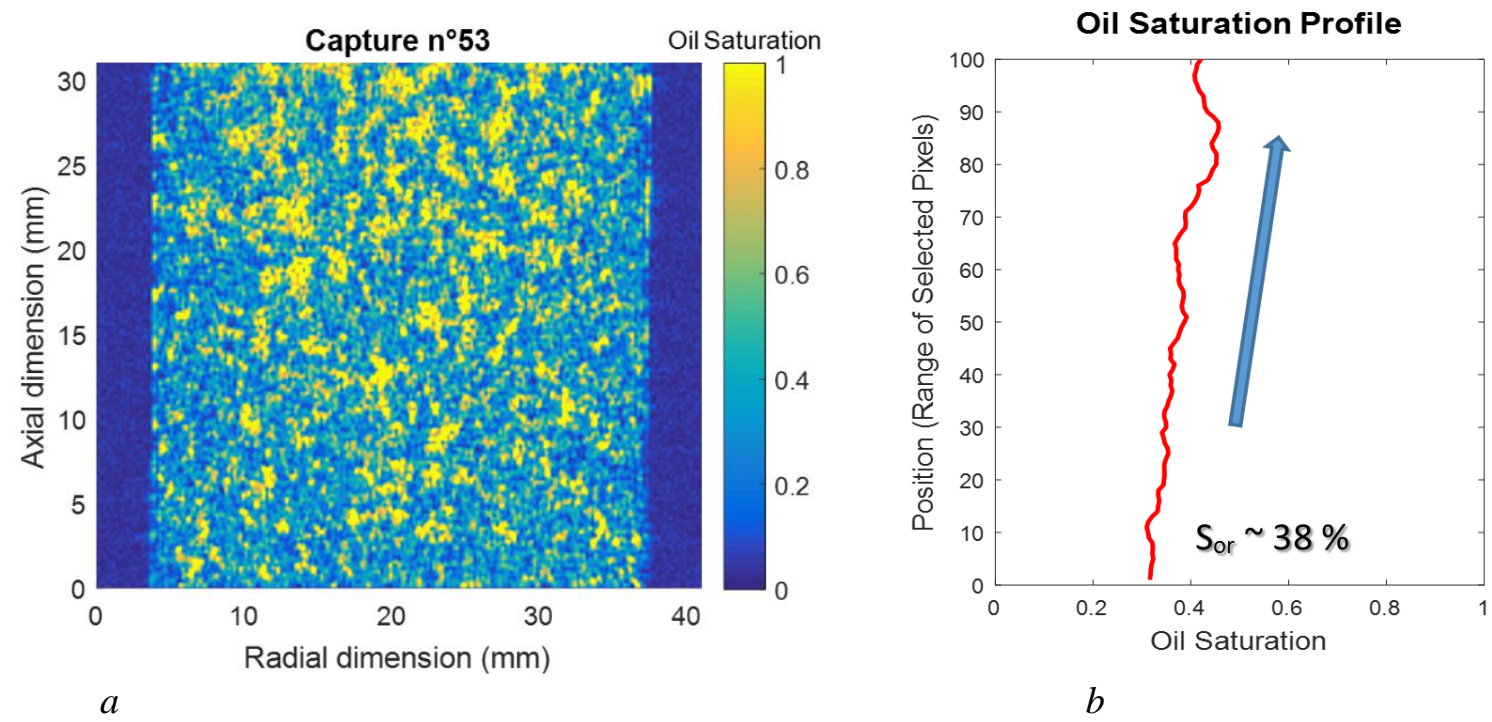

Figure 4: Residual oil saturation image (a) and corresponding saturation profile (b) at the end of the displacement process after the injection of 3.5 PV of the displacing fluid.

For this test two types of residual oil saturation were observed. The first type corresponds to the large oil droplets grouped into small discontinuous formations elongated in the direction of flow; the second type of residual oil corresponds to the oil films, which cover the oil-wet polystyrene beads.

The visualization and statistical analysis of this experimental test show that from a total residual oil saturation of $38 \%$ only $14 \%$ is trapped due to development of digitations and the capillary pressure. It can be seen that a large part of the residual oil saturation (estimated as 24\%) consists of the oil films on the surface of the polystyrene beads due to the wettability effect (oil wet material).

\section{Experiment with packed sand column}

The experiments on polystyrene and borosilicate beads have shown the clear internal structure of porous model and the saturation distribution of phases in space and time. It was even possible to see the beads of $0.6 \mathrm{~mm}$ diameter with the spatial resolution of MRI tool of $0.19 \mathrm{~mm}$ for 1 pixel. Unfortunately, this size of grains of porous medium does not allow for the capillary forces to be important because of the big size of the pores. To study the simultaneous effect of the viscous, capillary and gravity forces, we have to reduce the grain size and also use some particle-size distribution. For this purpose, we used silica sand.

Table 2 shows the main parameters of the experimental investigation with the packed sand model. The concentration of the manganese chloride was increased two times because of the adsorption capacity of the sands.

\begin{tabular}{ll}
\hline \multicolumn{2}{l}{ Table 2. Water flooding experiment in packed sand } \\
\hline Porous & Packed sand of particle size $<0.16 \mathrm{~mm}$ \\
\hline Tube & Glass tube of $10 \mathrm{~cm}$ net length \\
\hline Injection rate & $0.06 \mathrm{ml} / \mathrm{min}$ \\
\hline Estimated porosity & $39 \%$ \\
\hline Injected fluid & Pure water $+\mathrm{MnCl}_{2}(\mathrm{C}=0.02 \mathrm{~mole} / \mathrm{l})$ \\
Oil phase (kerosene) & PTX2000 $\rho=800 \mathrm{~kg} / \mathrm{m} 3, \mu=2.96 \mathrm{mPa}$ at $20^{\circ} \mathrm{C}$ \\
MRI Field of view & $5 \mathrm{~cm}(256 \times 256$ pixels $)$ \\
\hline
\end{tabular}

ECMOR XVI $2018-16^{\text {th }}$ European Conference on the Mathematics of Oil Recovery 3-6 September 2018, Barcelona, Spain 
Firstly, silica sand was tested with the MRI spectrometer to see its impact on the signal and it was accepted as a good material. Then, a particle-size distribution was made with a grains size distribution from $0.05 \mathrm{~mm}$ to $0.16 \mathrm{~mm}$. Finally, the sand in the model was compacted using a vibrating table, and an estimation of the porosity and the permeability was done.

The oil extraction by water flooding in the packed sand model was performed with a slow injection rate of $0.06 \mathrm{ml} / \mathrm{min}$. The estimation of the dimensionless parameters in this process gives the following: Capillary number Ca 1.e-4 and Bond number Bo 0.1, which confirms the important effect of the capillary phenomena. This experiment (Fig.5) has shown that the advance of the displacement front happens in the form of occasional jumps and is controlled by the gravity, viscous and capillary forces. In spite of the fact that under the vertical direction of flow the viscous instability is partially compensated by the gravity and capillary forces, the displacing phase forms fingerings.

A series of twelve consecutive images with an interval of 15 minutes is shown in Figure 5. The appearance and the growth of fingers can be clearly observed in these pictures. The first fingers appear in the local micro imperfections of the sand model. Then, they slowly grow in space with help of the capillary forces. The next break-through of the fingering happens only when the water saturation on the front reaches some sufficient value. New fingers then appear and start to grow. It was observed, that there is an alternation between the capillary penetration and the appearance of a new viscous finger. The time between these alternations depends on the injection rate and model permeability.
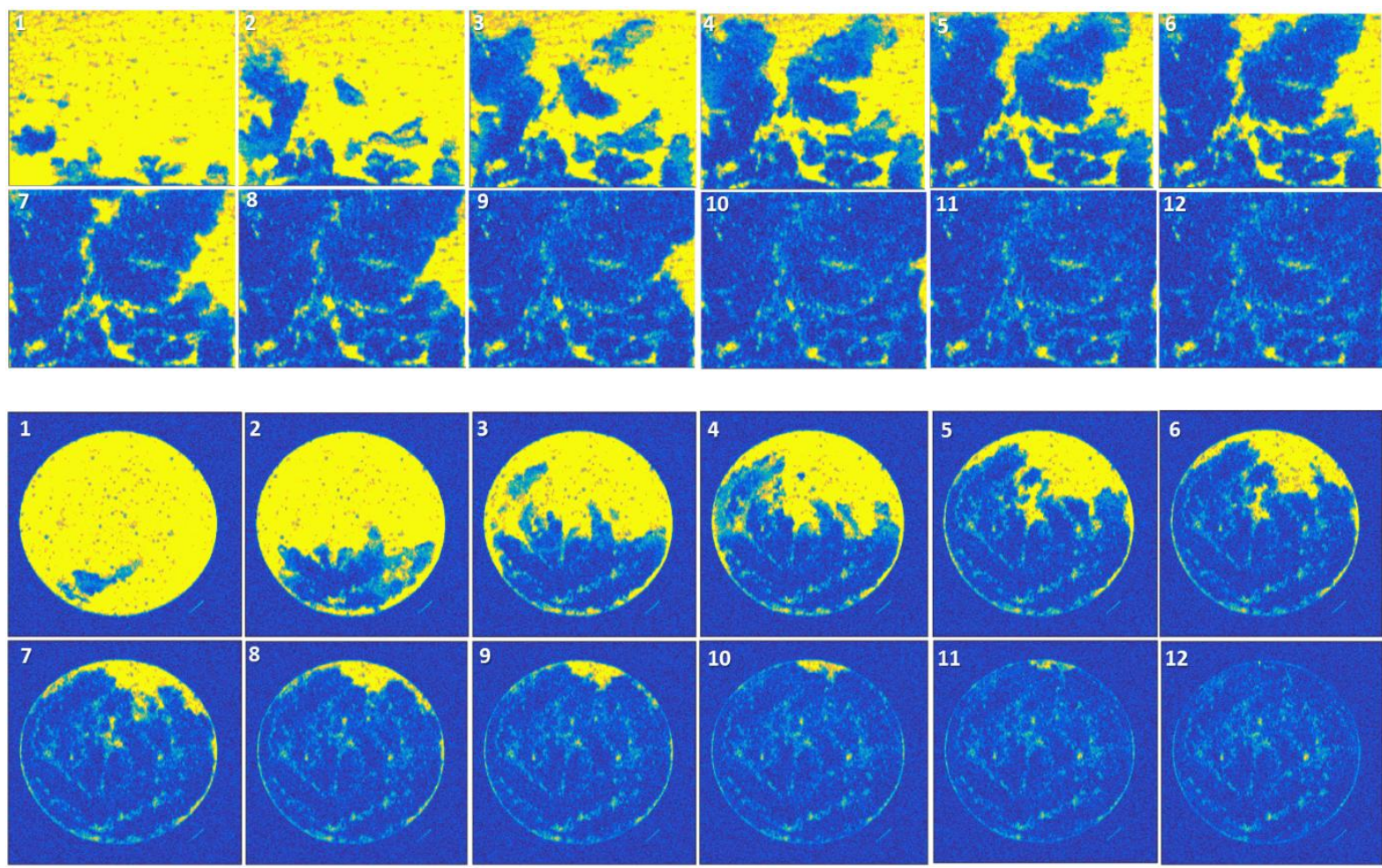

Figure 5: Consecutive MRI images of the water-oil displacement in the model of packed sand column with the injection flow rate of $0.06 \mathrm{ml} / \mathrm{min}$. The time interval between each image equals 15 minutes.

The vertical and horizontal cross-sections from Figure 5 show that the loss of the connectivity of oil phase between the water fingers leads to the formation of the trapped oil. For the given experiment the final residual oil saturation after injection of $3 \mathrm{PV}$ of water equals to $15.2 \%$. 
The oil saturation profiles were constructed from the MRI signal for every time of acquisition. We present in Figure 6 an intermediate image after $2 \mathrm{~h}$ of water injection. The detailed observation of this image shows the water saturation distribution in the model. The big volumes have a black color, which corresponds to high water saturation. However, the limits of these fingers have a light grey color, which corresponds to a lower saturation of water and a penetration of water by the capillary effects. Figure 6 also shows the saturation profiles of oil phase, where the dashed red line corresponds to the selected image.

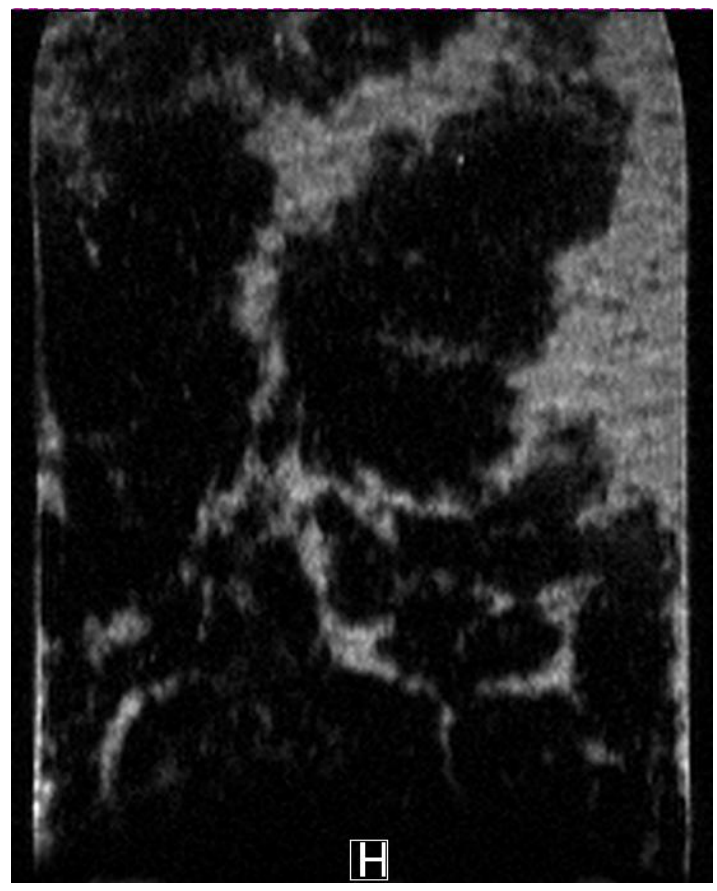

$a$

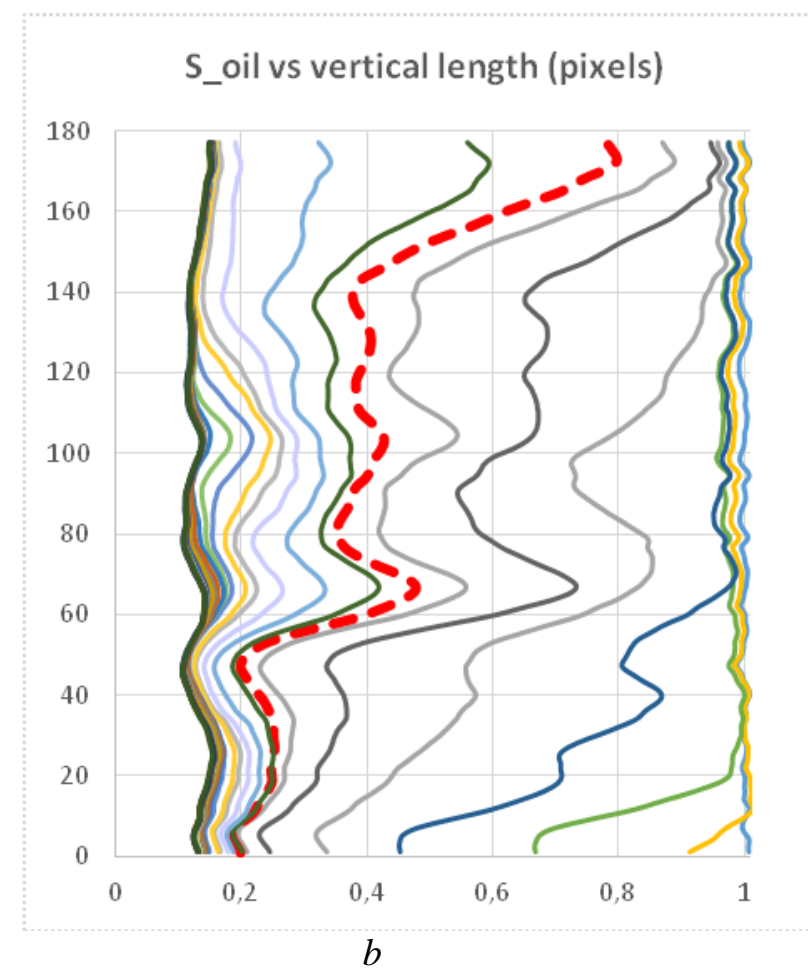

$b$

Figure 6: Oil saturation distribution in the packed sand model, the injection flow rate is $0.06 \mathrm{ml} / \mathrm{min}$ : (a) MRI image of displacement process after $2 h$ of injection, $(b)$ variation of oil saturation vs vertical coordinate in time, red dashed line corresponds to the saturation profile for the image (a).

The averaged oil saturation was calculated through the whole sample and is presented in Figure 7. The stabilization of the oil saturation in the model was observed after the injection of 2 pore volumes of the displacing phase.

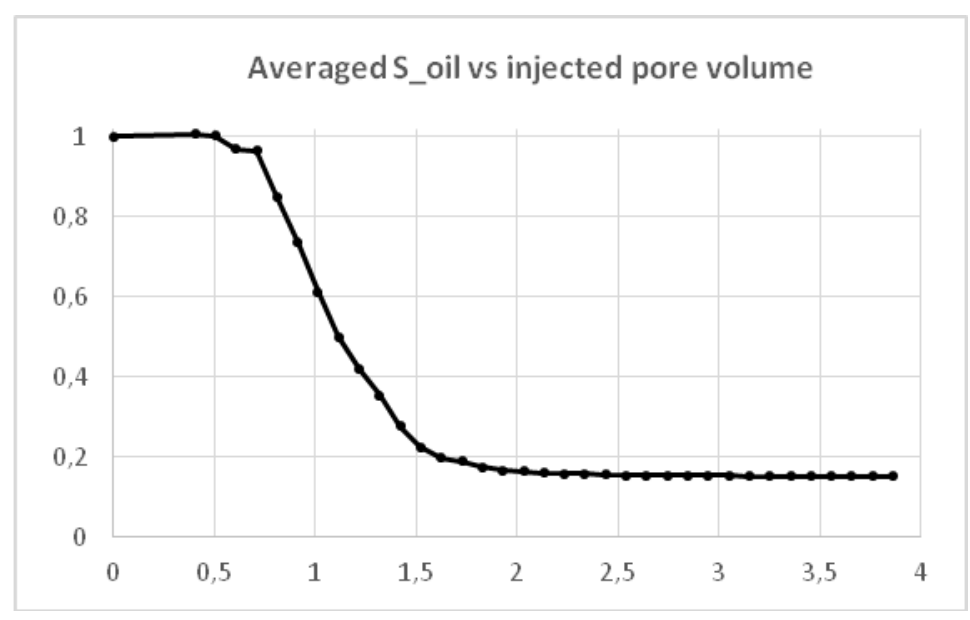


Figure 7: Averaged oil saturation vs injected $P V$ for the model of packed sand, $Q=0.06 \mathrm{ml} / \mathrm{min}$.

To better understand the mechanism of the pulsating movements observed on the condition of slow water pumping, a series of pictures is given in Figure 8.
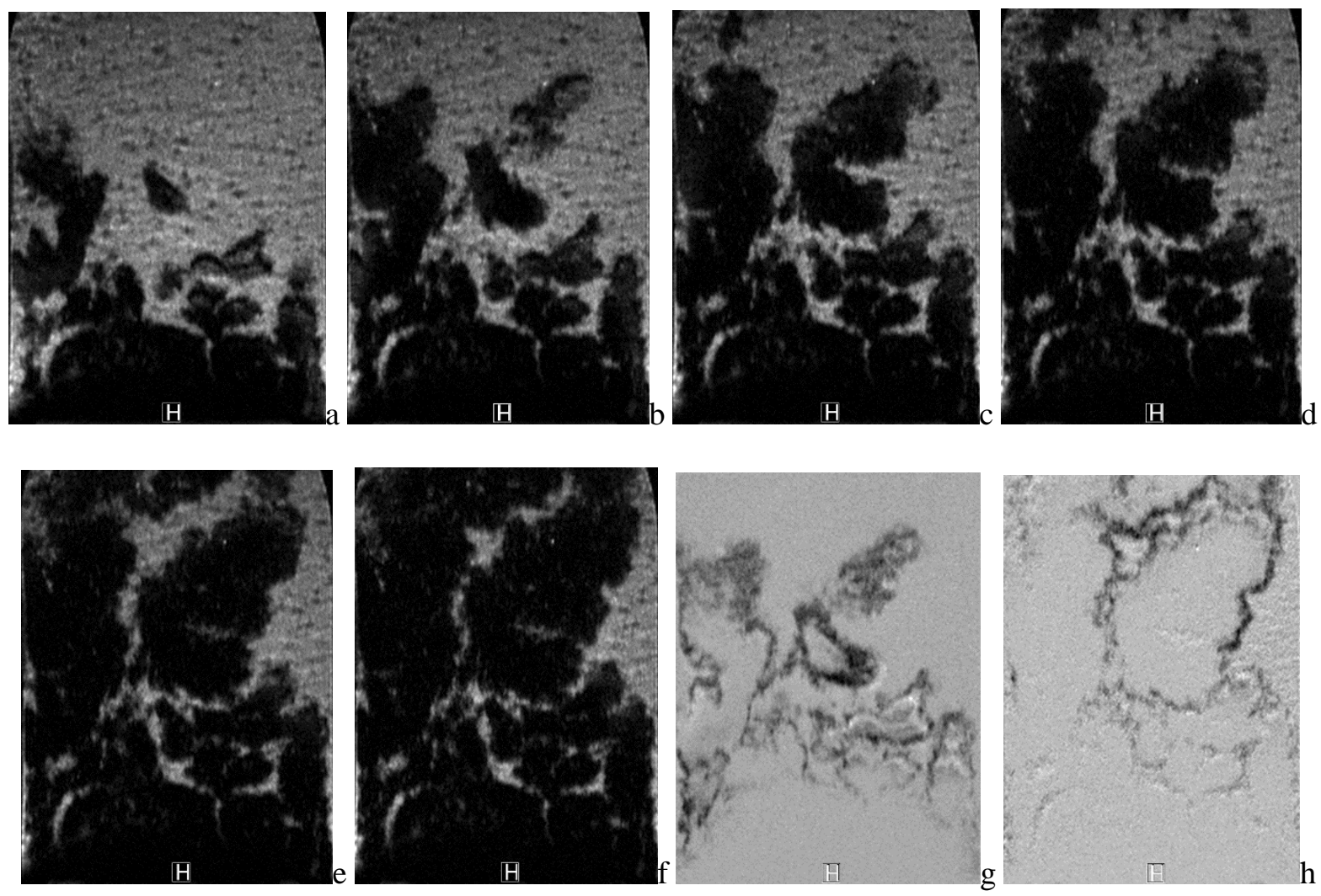

Figure 8: Oil-water displacement in the sand model, $Q=60 \mu \mathrm{l} / \mathrm{min}$ :

(a)-(f) consecutive images of fingers growth with the interval of 15 min; difference in oil saturation between the stages $a-b=(g)$ and $e-f=(h)$, which shows the local area where the displacement happens.

The difference between the signals of two consecutives images highlights the evolution of the two phases. Figure 8(g) corresponds to the difference of the images (a) and (b). Here, the intensity of the grey color shows the part of the front where the changes in the saturation happen. Generally it occurs on the perimeter of the larger fingers. The areas filled with the grey-black color correspond to the emergence of a new finger.

Similar experiments on sand were made with higher and lower flow rates. The observed process was similar, with difference in the size of the fingers and the interval of alternation with the capillary penetration. For the performed experiments the profiles of saturation and their variation with time were constructed. The statistical analysis of the residual oil saturation distribution was also made.

\section{Displacement problem formulation}

To model the process of the two-phase displacement, we used firstly the classical approach of Buckley-Leverett. We consider the two-phase immiscible system of fluids.

$$
\begin{aligned}
& \frac{\partial}{\partial t}\left(\rho_{i} \cdot S_{i} \times 0+\nabla\left(\rho_{i} \cdot V_{i}\right)=\mathbf{0}\right. \\
& V_{i}=-\frac{K k_{r i}}{\mu_{i}} \operatorname{grad}\left(P_{i}+\rho_{i} g z\right)
\end{aligned}
$$




$$
P_{c}=P_{o}-P_{w}
$$

where $i$ is the index of phase, namely $i=0, \mathrm{w} ; \rho$ is the density; $\mathrm{S}$ is the saturation; $\phi$ is the porosity; $\mathrm{V}$ is the Darcy velocity; $\mu$ is the dynamic viscosity; $\mathrm{P}$ is the pressure; $\mathrm{K}$ is the intrinsic permeability of porous medium; $k_{r}$ is the relative permeability; $\mathrm{g}$ is the gravitational acceleration; $\mathrm{z}$ corresponds to the vertical coordinate. The capillary pressure $P_{c}$ at any point of the porous medium denotes the difference between the $P_{o}$ and $P_{w}$.

The classical Buckley-Leverett approach does not consider the variation of fluid density, $\rho_{i}=$ const . This hypothesis can be accepted in our case for the displacement process of oil by water at the ambient conditions.

The system (1)-(3) can be transformed into one equation for water saturation $S$ :

$$
\left(\partial S / \partial \mathbf{t}+\nabla\left(F(S) V_{\downarrow} t o t-F(S) \lambda_{1} o g\left(\rho_{\downarrow} w-\rho_{1} o\right)+F(S) \lambda_{1} o \operatorname{grad} P_{\downarrow} c\right)=0\right.
$$

where $S$ is the displacing phase saturation; $V_{t o t}=V_{w}+V_{o}$ is the total velocity; $\lambda_{i}=\frac{K k_{r i}\left(S_{i}\right)}{\mu_{i}}$ describes the $i$-phase mobility.

The function $F(S)$ defines a fraction of the displacing phase flux in the total flux neglecting the gravity and the capillary forces:

$$
F(S)=V_{\downarrow} w / V_{\downarrow} \text { tot }=\left({ }_{1} w /\left(\mathrm{G}_{1} o+G_{1} w\right)\right.
$$

The power-type relationships between the normalized saturation and the relative permeability are defined as:

$$
\left\{\begin{array}{c}
S\left(S_{w r}: 0\right. \\
S>S_{w r}: k_{r w}(S)=\left(\frac{S-S_{w r}}{1-S_{w r}}\right)^{2}
\end{array}\right.
$$$$
\left\{\begin{aligned}
S\left(S_{r o}: \boldsymbol{k}_{\text {or }}(\boldsymbol{S})\right. & =\left(\frac{1-S-S_{\text {or }}}{1-S_{\text {or }}}\right)^{2} \\
S>S_{\text {or }}: & \mathbf{0}
\end{aligned}\right.
$$

At the initial state the model is completely filled with oil, so $S_{\text {init }}=\mathbf{0}$. The boundary conditions for the equation (4) are: $S=1$ at the bottom and the constant flux at the top of the model.

The classical model of the two-phase flow takes the capillary effects only as the dispersion phenomena of displacing saturation. The phenomenological model with vector nature of capillarity was proposed in (Panfilova, Panfilov, 2005). To describe the phase distribution this model demands two specific parameters: the volume fraction of the mobile displacing phase in the area of interface between the phases (1), and the degree of connectivity between them (2). Basing on the experimental results and the properties of the internal structure of phase distributions in the model we propose closing relations for the vector model and compare it with the classical approach.

For the model with the vector nature of capillary forces the equation (4) takes form:

$$
\varphi \frac{\partial S_{w}}{\partial \mathrm{t}}+\boldsymbol{\nabla}\left(F(S) V_{t o t}-F(S) \lambda_{o} g\left(\rho_{w}-\rho_{o}\right)+F(S) \lambda_{o} \gamma \eta_{c} \frac{P_{c}}{l} \overrightarrow{e_{c}}\right)=\mathbf{0}
$$


where $Y$ is the sign of the acting of the capillary forces, which depends on the wettability of the phases; $\boldsymbol{\eta}_{\boldsymbol{c}}$ is the specific concentration in meniscus or volume fraction of mobile wetting phase in the area of interface; $\boldsymbol{l}$ is the characteristic pore size; $\overrightarrow{\vec{e}_{c}}$ is the unit vector of capillary force.

\section{Numerical modelling}

The numerical simulations were made for an axisymmetric 3D model. The geometrical parameters and the properties of fluids were taken from the experimental investigation set for the packed sand. For this medium the water is the more wetting fluid. The initial saturation of water equals to zero. To complete the relations (6) for the saturation functions, the residual saturations of phases were taken as follows: $S_{o r}=0.25$ and $S_{w r}=0.15$. The capillary pressure $P_{c}(S)$ was constructed using the Jfunction from the Leverett experiments for the non-consolidated porous materials.

Figure 9 shows the solution of classical formulation, where 3 forces are taken in consideration, namely the gravity, capillary and viscous forces.

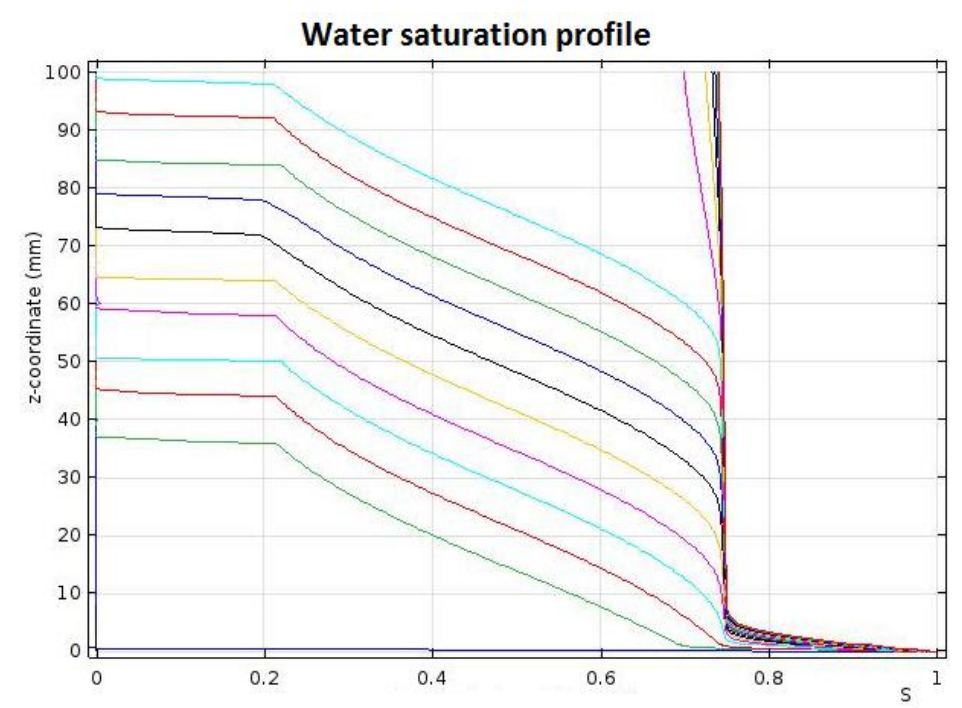

$a$

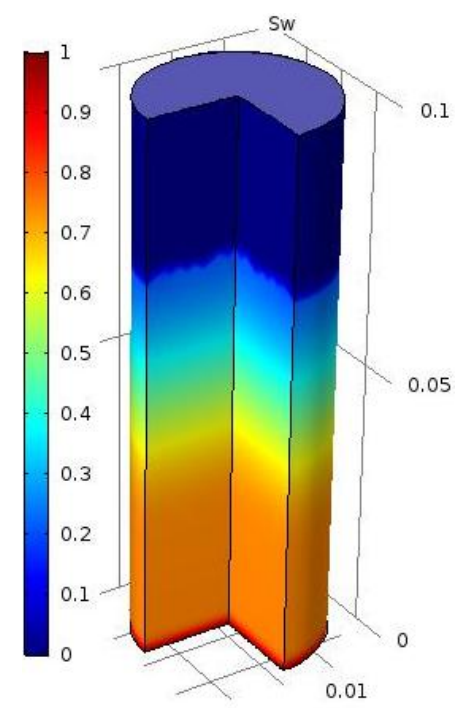

$b$

Figure 9: Water saturation profile vs vertical position (a) and saturation distribution in $3 D$ view (b).

The time of averaged front propagation in the numerical simulations corresponded well to the time of the process in the experimental study. The simulation results show that the oil saturation trapped in the model remains constant with time and equals to the micro trapped oil saturation $S_{\text {or }}$, Fig.9(a). It means that for our case the classical approach with the important influence of the gravity and capillary forces leads to the ideal recovery, without macro trapping of oil.

The comparison of the influence of three main forces was made in Figure 10. The oil saturation profile for the time of water injection 1.5h was calculated for three cases: classical Buckley-Leverett model (curve 1), classical model with gravity effects (curve 2) and finally the Buckley-Leverett model with gravity and capillary phenomena (curve 3). It is evident that the gravity forces slow down the front propagation in the model, but they also stabilize the displacement process. The capillarity effects accelerate the front propagation mostly for the small water saturations, where the capillary pressure is maximum. Following these results, the gravity and capillary phenomena lead to a good sweep efficiency and does not take into account the macro oil trapping provoked by the viscous instability. 


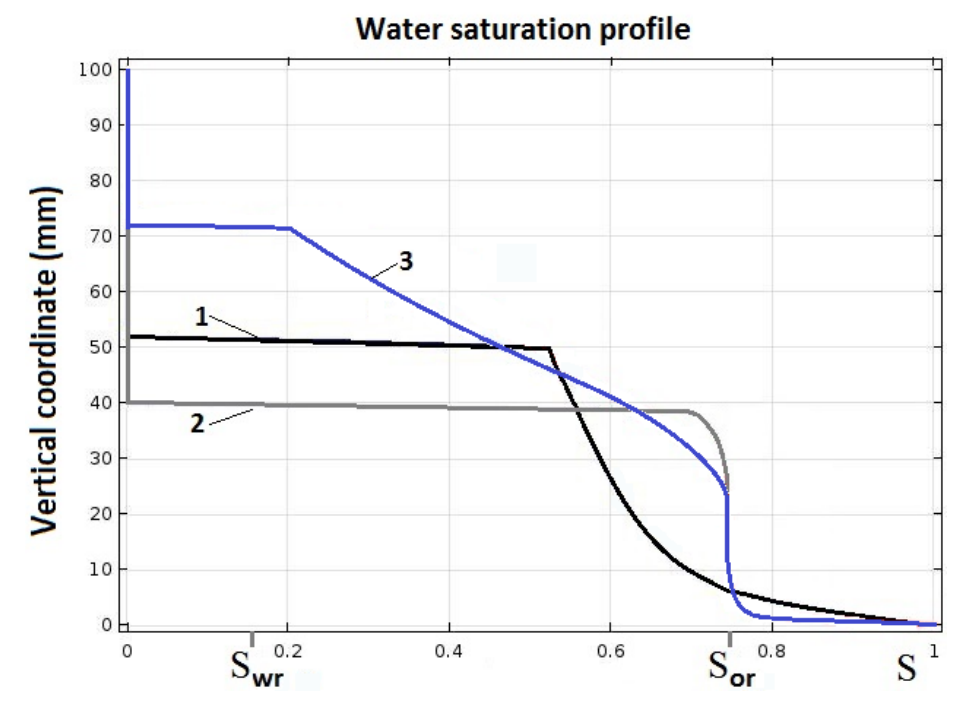

Figure 10: Water saturation profile vs vertical coordinate for 3 cases:

(1) classical BL model, (2) BL model with gravity, (3) BL model with gravity and capillarity.

The numerical simulation with the vector model show qualitatively similar results (Fig.11a). But here, the sharp shock observed for the classical model disappears with the capillarity effects. The simulation for the equation (7) used the specific parameter as the meniscus concentration in space or volume fraction of mobile wetting phase in the area of interface. This parameter was obtained by the analysis of the experimental results and is presented in Fig.11b. The mobile phase saturation was obtained by calculation of the saturation differences from the MRI images. The zone of residual oil saturation was removed because of the immobility of fronts in this area.
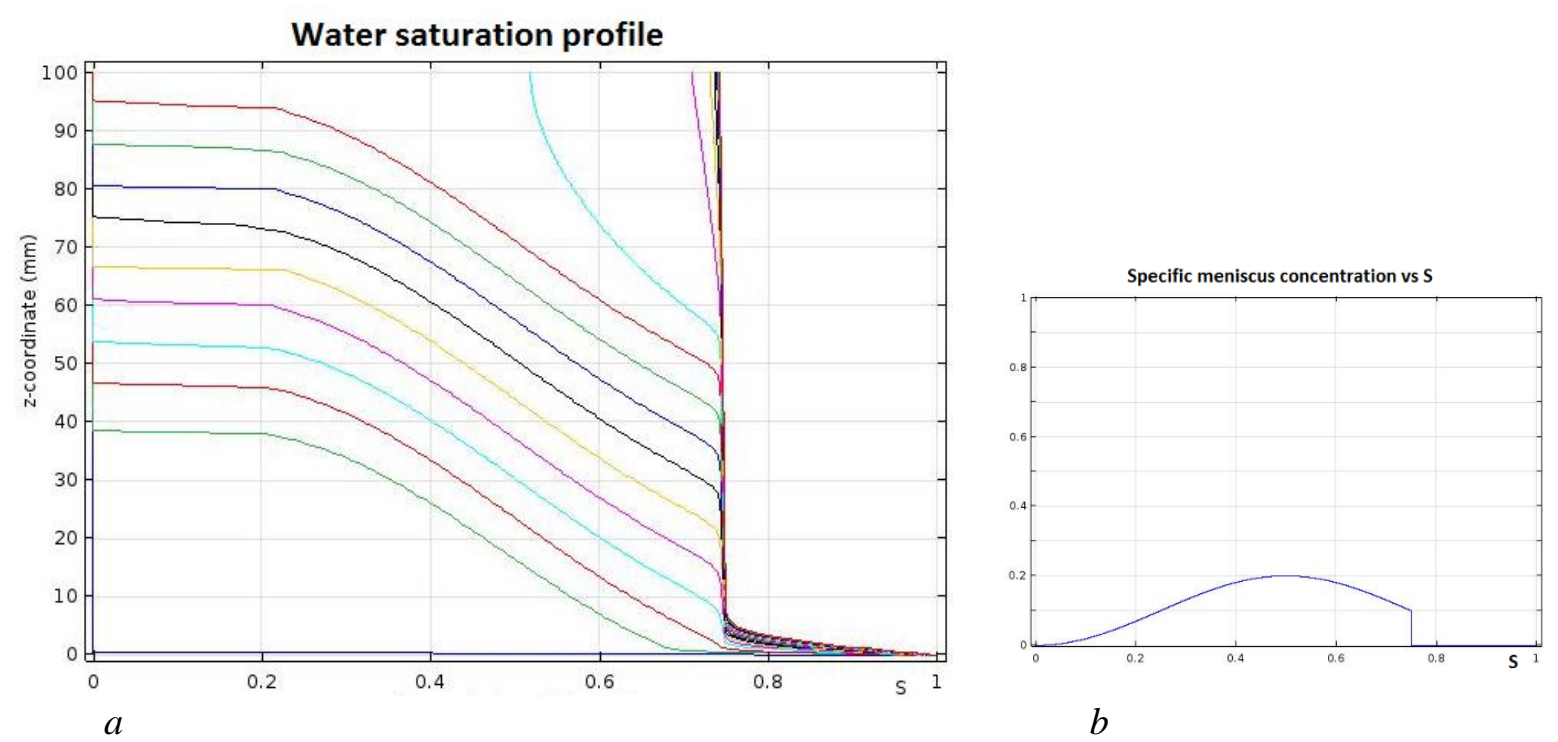

Figure 11: Water saturation profile vs vertical position for vector capillary model (a) and specific meniscus concentration as a function of saturation (b)

Finally, the comparison between the fluxes caused by three main forces was made in Figure 12. In Fig.12a, the fractional flow flux, the capillary flux and the gravity flux are shown separately to better understand the influence of each of them. 

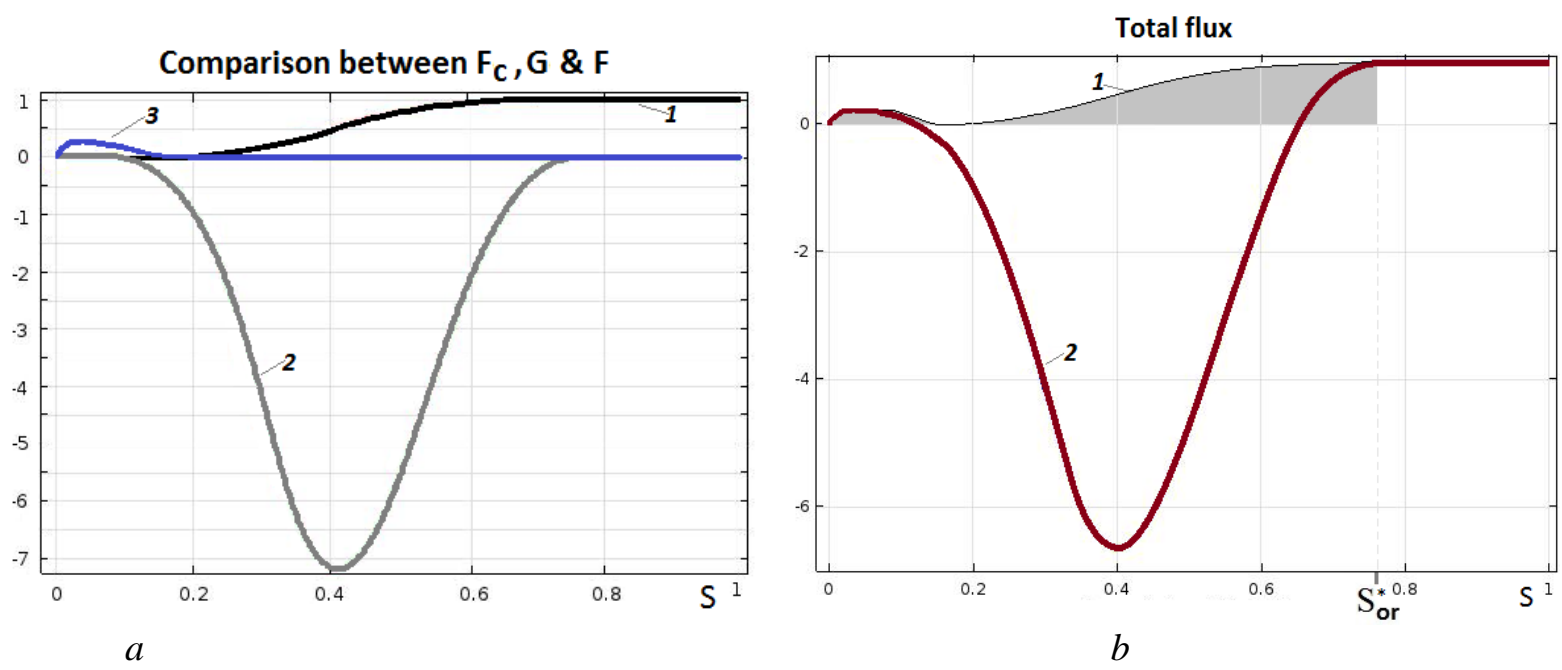

Figure 12: (a) - Fractional flow (1), gravitational (2) and capillary (3) fluxes for the eq.(7); (b) - Total flux (2), the sum of capillary and gravity flux (1), the grey color indicates the zone of the water saturation, where the front can move forward.

Figure 12 explains the behavior observed during the experimental investigations on the packed sand model. The fingers have grown under the capillary and gravity forces, but at the same time the propagation of water was blocked locally, and some part of the displacement front did not move for hours. Then, the situation of immobility was changed by the collapse with another finger or by gravity segregation. This partial immobility can be explained by the following: when the gravity is compensated by the water pumping, only the saturation with non-zero mobility can move. The area of mobile water saturation is marked in grey color in Figure $12 \mathrm{~b}$. It means that there is some interval of saturation which can flow only by gravity, because this saturation is lower than the residual water saturation and greater than the saturation which corresponds to the positive non-zero capillary flux.

Figure 12 also shows that the capillary effects are important only for the small water saturation; for the other values of saturation the capillary effects are negligible in comparison with gravity and viscous effects.

\section{Conclusions}

High-resolution MRI technique was used to investigate two phase fluids flow of oil and water in porous media. The porous media was formed from packed polystyrene beads or sand. The importance of water channeling or fingering phenomena was obviously found. Evidently, the size of the beads/grains and the value of porosity play an important role in the displacement front deformation.

Positive impact was observed by adding $\mathrm{MnCl} 2$ to the injected water phase. It has shortened the $\mathrm{T}_{2}$ of water relaxation time to $0.7 \mathrm{~ms}$ instead of $2.35 \mathrm{~s}$ without any effect on the $\mathrm{T}_{2}$ of the oil phase.

The numerical simulations, which take into consideration the phase distribution in porous medium, the displacing front deformation and the residual phase formation, were made. The closing relations for the two-phase vector model were obtained from the given experiments. The simulations confirmed qualitatively the experimental results. 


\section{Acknowledgments}

This work is supported by two laboratories LEMTA (Laboratoire d'Energétique et de Mécanique Théorique et Appliquée, UMR 7563) and CRM2 (Laboratoire de Cristallographie, Résonance Magnétique et Modélisations, UMR 7036), and partially financed by TOTAL.

The authors are grateful to the master students of University of Lorraine: J.Alakbarli, L.Mancini and D.L.Zadi.

\section{References}

Bloembergen, N. [1957] Proton relaxation times in paramagnetic solutions. Journal of Chemical Physics, 27, 572-573.

Erslanda, G., Fernøa, M.A., A. Grauea, Baldwinb, B.A., Stevensc, J. [2010] Complementary imaging of oil recovery mechanisms in fractured reservoirs, Chemical Engineering Journal, 158, 32-38.

Johns, M. L. and Gladden, L.F. [1999] Magnetic resonance imaging study of the dissolution kinetics of octanol in porous media, Colloid and Interface Science, 210, 261-270.

Mitchell, J., Chandrasekera, T.C., Holland, D.J., Gladden, L.F., Fordham, E.J. [2013] Magnetic resonance imaging in laboratory petrophysical core analysis, Physics Reports, 526, 165-225.

Panfilova, I. and Panfilov, M. [2005] Phenomenological meniscus model for two-phase flow through porous media. Transport in Porous Media, 58(1), 87-119.

Salameh, W., Leclerc, S., Stemmelen, D., Escanyé, J.M. [2010] NMR imaging of water flow in packed beds. Diffusion Fundamentals, 14(5) 1-5. 